\title{
Simultaneous Imaging of Cryo-Bright Field, Dark Field STEM and SEM Using Unroofed Living Cells with Special Reference to Membrane Cytoskeletons.
}

\author{
Jiro Usukura and Shiho Minakata
}

Division of Integrated Research Projects, EcoTopia Science Institute, Nagoya University, Nagoya Japan

Cryo-electron microscopy has been used exclusively so far for single particle analysis or crystallography of purified proteins. Recently, technical improvements on rapid freezing and cryo-microtome permitted structural analysis of organelle or macromolecules in native state using whole cells $(1,2)$ and vitreous cryo-sections (3). However, cryo-sections don't scatter electrons so much and therefore show quite low contrast in addition to weakness against electron irradiation. They complicate a searching and a focusing of samples. In order to overcome these difficulties, we are improving unroofing techniques for preparation of cryo-electron microscopy of cells together with development of SEM based cryo-STEM. We succeeded simultaneous imaging of cryo-dark field and bright field STEM and secondary electron with extremely high contrast and accuracy for untreated cells.

Procedures: HeLa or ECV cells were cultured on the molybdenum mesh grid covered with carbon coated polyvinyl Formvar membrane for a few days in DMEM. Cultured cells were washed once with HEPE based Ringer's solution and placed in KHMgE buffer (30 mM HEPES, pH 7.4, 70 mM KCl, 3 $\mathrm{mM} \mathrm{MgCl}$, $1 \mathrm{mM}$ EGTA, $1 \mathrm{mM}$ DTT, $0.1 \mathrm{mM}$ AEBSF (4-(2-aminoethyl) benzenesulfonyl fluoride hydrochloride)). Subsequently, dorsal cell membrane was removed partially by weak sonication induced buble jet with meticulous care (unroofing). Cells unroofed were washed briefly in fresh KHMgE buffer and then quickly frozen by plunging them into liquid ethane using Leica EM GP rapid freezing apparatus. Frozen samples were then brought into SU9000 scanning electron microscope (Hitachi High-Technologies Co.) equipped with improved anti-contamination trap by using newly developed cryo-transfer holder.

Results and Discussion: Simultaneous imaging of cryo-dark field, bright field STEM and secondary electron (SE) was very useful for evaluating sample condition and judging the irradiation damages. For example, if anti-contamination trap is incapable of absorbing water vapor enough, mesh grids became frosted during observation. Comparison the STEM images with SEM images elucidated obviously that such frosting occurred always underside of the grid. SEM also was capable of visualizing evaporation extent induced by electron irradiation as the undulation of ice embedding samples. That is, it was measured how much the sample received irradiation damages while observing. Although the unroofing technique employed in preparation procedure was developed originally for observing membrane undercoat in freeze-etching replica method (4), it was splendid technique that could be applied to atomic force microscopic (AFM) imaging (5). We improved and extended this technique further for cryo-electron microscopy. Soluble proteins in cytoplasm flew out on removing the part of cell membrane mechanically (unroofing). Cytoskeleton and structural proteins are remained in situ, so that structural proteins become observable with enough contrast. As expected, membrane cytoskeleton composed of actin filaments and microtubules were found on the cytoplasmic surface of cell membrane with extremely high contrast. In the cortical area, the filaments often extended in parallel, as shown in Fig.1. In other regions, however, fine filaments extended in various directions on the membrane while aggregating and dispersing at several points, which eventually divided the membrane surface into 
several domains (Fig. 2). The images obtained from samples prepared in this way must therefore be comparable between different observation methods. Confirmation of results by two different observation methods is very important for determining real structures. In general, In this case, cryo-electron microscopy imaging can be directly compared to freeze-etching electron microscopy by using the unroofing preparation method. Hopefully, these different imaging techniques can provide complementary data to characterize membrane cytoskeleton.

Conclusion: We succeeded simultaneous imaging of cryo-dark field, bright field STEM and SE, which was extremely useful for searching, evaluating and focusing of frozen cells. Actin filaments extending on the cell membrane with aggregation and dispaersion were visualized clearly in native state. Unroofing appeared to be better than vitreous cryo-sections on contrast and analysis of interaction between membrane surface and the filaments.

\section{References:}

[1] Medalia O, Weber I, Frangakis AS, Nicastro D, Gerish G, Baumeister W. Science 298 (2002) 1209-1213

[2] Kumer J, Frangakis AS, Baumeister W. Science 307 (2005) 436-438

[3] Pierson J, Vos M, Macintosh JR, Peters PJ J. Electron Microsc. 60 suppl 1 (2011): 93-100

[4] Heuser, J E, Anderson, R G J. Cell Biol. 108 (1989), 389-400

[5] Usukura J, Yoshimura A, Minakata S, Youn D, Ahn J, Cho SJ J. Electron Microsc. 61(2012), 321-326

[6] The author(J.U) acknowledges funding from the Japan Science and Technology Agency (JST), "Development of Systems and Technologies for Advanced Measurement and Analysis" Program.
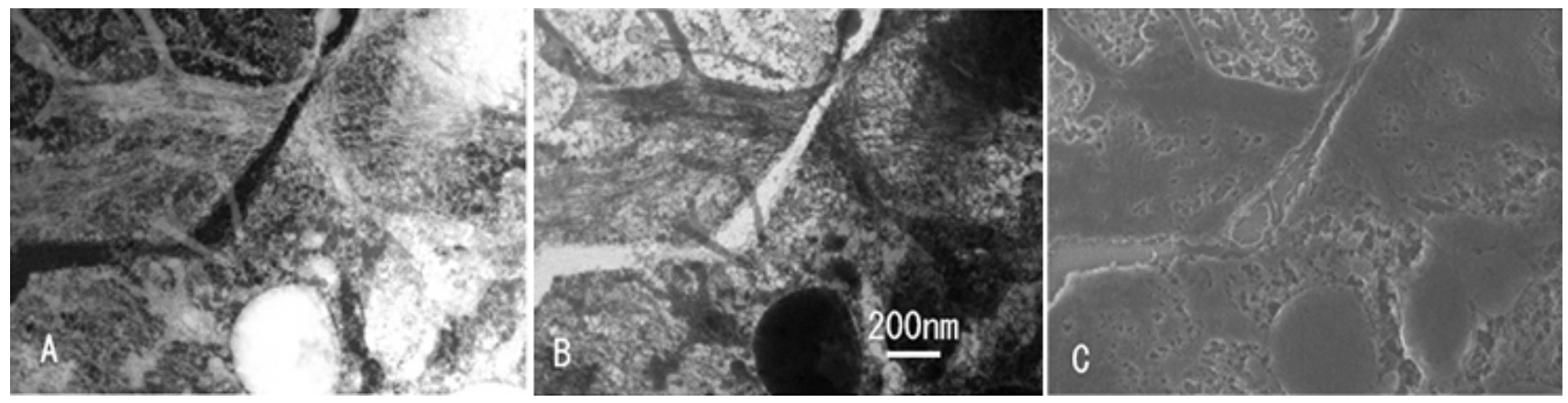

Figure 1. Simultaneous imaging of cryo-bright field (A), dark field (B) and SE (C). This sample received slightly irradiation damage.

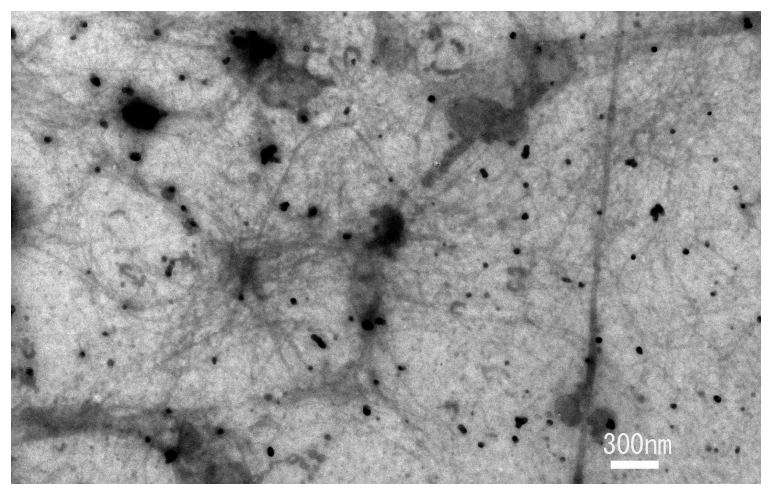

Figure 2. Cryo-bright field STEM image showing actin filaments network of membrane undercoat. 